\title{
Kaon semileptonic decay from the SU(3)-symmetric point down to physical quark masses
}

\author{
A. Jüttner*, J.M. Flynn, C.T. Sachrajda \\ School of Physics and Astronomy, University of Southampton, Southampton SO17 1BJ, UK \\ E-mail: juettneresoton.ac.uk, jflynnesoton.ac.uk, ctsesoton.ac.uk
}

\section{P.A. Boyle}

School of Physics \& Astronomy, University of Edinburgh, EH9 3JZ, UK

E-mail: paboyleeph.ed.ac.uk

\section{R.D. Mawhinney, H. Yin}

Physics Department, Columbia University, New York, NY 10027, USA

E-mail: rdmephysics.columbia.edu, yinnhtephys.columbia.edu

\section{N. Garron}

School of Mathematics, Trinity College, Dublin 2, Ireland

E-mail: ngarron@maths.tcd.ie

\section{K. Sivalingam}

Department of Meteorology, University of Reading, Reading, RG6 6BB, UK

E-mail: k.sivalingamereading.ac.uk

\begin{abstract}
We present an update on the RBC/UKQCD collaboration's results for the $K \rightarrow \pi$ semileptonic vector form factor at zero momentum transfer from simulations of chiral fermions. Our results cover the whole range of light quark masses between the $\mathrm{SU}(3)$-symmetric and the physical point for three lattice spacings and large physical volumes. Using partially twisted boundary conditions we calculate the form factor directly at zero momentum transfer. The comprehensive set of data points allows for turning the extrapolation in the quark mass into an interpolation around the physical point thereby removing the dominant systematic uncertainty in previous results, the chiral extrapolation. We briefly discuss our prediction in view of Standard Model phenomenology.
\end{abstract}

31st International Symposium on Lattice Field Theory - LATTICE 2013

July 29 - August 3, 2013

Mainz, Germany

\footnotetext{
${ }^{*}$ Speaker.
} 


\section{Introduction}

The a priori unknown unitary Cabibbo-Kobayashi-Maskawa (CKM) matrix [1,2] parameterises flavour changing processes and CP-violation in the Standard Model (SM). By combining precise and reliable theory predictions and experimental measurements one is overconstraining its elements in the search of tiny inconsistencies which would hint towards extensions of the SM, i.e. new physics. This talk reports on our continued efforts $[3,4,5]$ in the computation of the $K \rightarrow \pi$ semileptonic vector form factor in $N_{f}=2+1$ Domain Wall lattice QCD at zero momentum transfer. The form factor at this kinematical point is a crucial ingredient in the extraction of the CKM-matrix element $\left|V_{u s}\right|$ [6]. Simulations of lattice QCD are the only known tool allowing for the prediction of the form factor from first principles and in a systematically improvable fashion. We note that by now the determination of $\left|V_{u s}\right|$ from leptonic kaon (and pion) decays has reached the same level of precision as the one we focus on in this talk (cf. [6]).

\section{Strategy}

The product $\left|V_{u s}\right|^{2}\left|f_{+}(0)\right|^{2}$ can be determined with a precision of around $0.2 \%$ from the experimental decay rate [7], and correspondingly a precise determination of the $K \rightarrow \pi$ vector form factor $f_{+}^{K \pi}(0)$ allows for the determination of $\left|V_{u s}\right|$. The matrix element

$$
\left.\left\langle\pi\left(p_{\pi}\right)\left|V_{\mu}\right| K\left(p_{K}\right)\right\rangle\right|_{q^{2}=0}=f_{+}^{K \pi}(0)\left(p_{\pi}+p_{K}\right)_{\mu}+f_{-}^{K \pi}(0)\left(p_{\pi}-p_{K}\right)_{\mu},
$$

of the flavour-changing vector current $V_{\mu}=\bar{u} \gamma_{\mu} s$ at vanishing momentum transfer $q^{2}=\left(p_{K}-p_{\pi}\right)^{2}$ can be extracted from the Euclidean time dependence of suitably chosen ratios of meson three point functions [8], e.g.,

$$
R_{\mu, K \pi}^{(2)}\left(t_{\mathrm{snk}}, \vec{p}_{K}, \vec{p}_{\pi}\right)=2 \sqrt{E_{K} E_{\pi}} \sqrt{\frac{C_{\mu, K \pi}\left(t, t_{\mathrm{snk}}, \vec{p}_{K}, \vec{p}_{\pi}\right) C_{\mu, \pi K}\left(t, t_{\mathrm{snk}}, \vec{p}_{\pi}, \vec{p}_{K}\right)}{C_{0, K K}\left(t, t_{\mathrm{snk}}, \vec{p}_{K}, \vec{p}_{K}\right) C_{0, \pi \pi}\left(t, t_{\mathrm{snk}}, \vec{p}_{\pi}, \vec{p}_{\pi}\right)}} .
$$

Here, $C_{\mu, P P^{\prime}}\left(t, t_{\mathrm{snk}}, \vec{p}_{P}, \vec{p}_{P^{\prime}}\right)$ is the three point function of the corresponding vector current $V_{\mu}(t)$ at Euclidean time $t$ (cf. [8] for more details). This ratio can be evaluated for all four components $\mu$ of the vector current and is constructed such that it equals (2.1) (for large Euclidean time-separations of the operator insertions). Note that the vector current renormalisation cancels between numerator and denominator. Alternatively we can also employ the Ward identity relating the vector current to the scalar current,

$$
\left.\left\langle\pi\left(p_{\pi}\right)|S| K\left(p_{K}\right)\right\rangle\right|_{q^{2}=0}=f_{0}^{K \pi}(0) \frac{m_{K}^{2}-m_{\pi}^{2}}{m_{s}-m_{u}},
$$

for which similar ratios of three point functions can be defined. Note that $f_{0}^{K \pi}(0)=f_{+}^{K \pi}(0)$ and that the renormalisation of the scalar current and the quark masses in the above relation cancel [9].

Due to the quantisation of quark- and therefore hadron-momenta in the finite lattice box the kinematical point $q^{2}=0$ is generally not accessible. We follow [10, 11, 12, 8, 4] and use partially twisted boundary conditions for the quark fields, $\psi(x+L \hat{i})=e^{i \theta_{i} / L} \psi(x)$, where $L$ is the spatial extent of the lattice, $i$ one of the spatial directions and $\psi$ one of the up-, down- and strange quark fields. By suitably choosing the quark's boundary conditions as a function of the pion and kaon masses [8] we achieve $q^{2}=0$ in all our simulations. 


\begin{tabular}{lllllll|ccccccc}
\hline \hline set & $\beta$ & $a / \mathrm{fm}$ & $L / a$ & $T / a$ & $m_{\pi} / \mathrm{MeV}$ & $m_{\pi} L$ & set & $\beta$ & $a / \mathrm{fm}$ & $L / a$ & $T / a$ & $m_{\pi} / \mathrm{MeV}$ & $m_{\pi} L$ \\
\hline $\mathrm{A}_{3}$ & 2.13 & 0.11 & 24 & 64 & 678 & 9.3 & $\mathrm{C}_{8}$ & 2.25 & 0.09 & 32 & 64 & 398 & 5.5 \\
$\mathrm{~A}_{2}$ & 2.13 & 0.11 & 24 & 64 & 563 & 7.7 & $\mathrm{C}_{6}$ & 2.25 & 0.09 & 32 & 64 & 349 & 4.8 \\
$\mathrm{~A}_{1}$ & 2.13 & 0.11 & 24 & 64 & 422 & 5.8 & $\mathrm{C}_{4}$ & 2.25 & 0.09 & 32 & 64 & 295 & 4.1 \\
$\mathrm{~A}_{5}^{4}$ & 2.13 & 0.11 & 24 & 64 & 334 & 4.6 & $\mathrm{C}_{\text {phys }}$ & 2.25 & 0.09 & 64 & 128 & 137 & 3.8 \\
$\mathrm{~A}_{5}^{3}$ & 2.13 & 0.11 & 24 & 64 & 334 & 4.6 & $\mathrm{~B}_{42}$ & 1.75 & 0.14 & 32 & 64 & 248 & 5.7 \\
$\mathrm{~A}_{\text {phys }}$ & 2.13 & 0.11 & 48 & 96 & 141 & 3.9 & $\mathrm{~B}_{1}$ & 1.75 & 0.14 & 32 & 64 & 171 & 3.9 \\
\hline \hline
\end{tabular}

Table 1: Basic parameters for all ensembles of gauge field configurations.

The determination of $f_{+}^{K \pi}(0)$ then proceeds as follows: For a given set of simulation parameters (cf. table 1) one chooses the momenta $\vec{p}_{K}=\vec{\theta}_{K} / L$ and $\vec{p}_{\pi}=\vec{\theta}_{\pi} / L$ for which the momentum transfer vanishes. One then computes the above ratios and thereby obtains an over-constrained system of linear equations (2.1) and (2.3) which one solves for $f_{+}^{K \pi}(0)$.

\section{Simulation parameters}

We simulate $N_{f}=2+1$ dynamical flavours of domain wall fermions covering the whole range of light quark masses from strange down to the physical light quark mass (for which the pion mass takes its experimental value). Our ensembles [13] comprise three different values of the lattice spacing and large volumes such that finite size effects should be well under control $\left(m_{\pi} L \gtrsim 4\right.$, cf. table 1). On the finer lattices we use the Iwasaki gauge action $[14,15]$ and the (Shamir/Möbius) domain wall fermion action $[16,17,18,19]$. Two of the ensembles have been generated with $\beta=$ 2.25 (labelled C), $a^{-1}=2.31(4) \mathrm{GeV}$ ) [20] and $\beta=2.13$ (labelled A), $a^{-1}=1.75(3) \mathrm{GeV}$ [21]. The coarser ensembles have been generated with the Iwasaki-DSDR action [20] at $\beta=1.75$ (labelled B), $a^{-1}=1.37(1) \mathrm{GeV}$.

\section{Data analysis and results}

In the following we present two distinct analyses: Analysis I has been published [5] and considers all ensembles except for $A_{\text {phys }}$ and $C_{\text {phys }}$ with physical light quark masses, analysing only (2.1) (cf. 1.h.s. plot in figure (1)). Analysis II is new and preliminary and comprises the full set of ensembles listed in table 1, analysing both (2.1) and (2.3) (r.h.s. plot in figure 1). This preliminary analysis is discussed in the next section. For analysis I we have studied three different ansätze for the mass-extrapolation:

$$
\begin{aligned}
& f_{+}^{K \pi}(0)=1+f_{2}\left(f, m_{\pi}^{2}, m_{K}^{2}, m_{\eta}^{2}\right)+\left(m_{K}^{2}-m_{\pi}^{2}\right)^{2}\left(A_{0}+A_{1}\left(m_{K}^{2}+m_{\pi}^{2}\right)\right), \\
& f_{+}^{K \pi}(0)=A+\frac{\left(m_{K}^{2}-m_{\pi}^{2}\right)^{2}}{m_{K}^{2}} A_{0}, \\
& f_{+}^{K \pi}(0)=1+\frac{\left(m_{K}^{2}-m_{\pi}^{2}\right)^{2}}{m_{K}^{2}}\left(A_{0}+A_{1}\left(m_{K}^{2}+m_{\pi}^{2}\right)\right) .
\end{aligned}
$$

The fits are motivated by the expectation for the mass dependence in chiral perturbation theory where $f_{+}^{K \pi}(0)=1+f_{2}\left(f, m_{\pi}^{2}, m_{K}^{2}\right)+\cdots$. In this NLO expression [22] the only unknown parameter is the decay constant $f$. We use this term and supplement it with higher order terms that respect 
SU(3) symmetry and are motivated by the complete NNLO computation [23]. We note that we do not have sufficient data to fit the full NNLO expression. The second ansatz is derived from a Taylor expansion of the NLO expression $1+f_{2}$ in the SU(3) breaking mass difference and in the third ansatz we supplement this expression by a model for higher order terms. Note that the second ansatz turns out to be rather stiff and unable to describe the curvature seen in the data close to the $\mathrm{SU}(3)$ symmetric point. By adding the free parameter $A$ we allow the fit to deviate from the SU(3) symmetric limit $f_{+}^{K \pi}(0)=1$. We find that all fits lead to mutually agreeing results when fitting only to the data closer to the physical point. This is illustrated in figure 2 which shows how the fit results behave under variation of the lightest (upper plot) and heaviest data point (lower plot) included into the fit.

Our final result is based on the third ansatz in (4.1) which we fit to all our data. As an estimate for the model-dependence we take the difference of the central value with respect to the central value of the fit with the first ansatz in (4.1) with $f=123 \mathrm{MeV}$. The final result is [5]

$$
f_{+}^{K \pi}(0)=0.9670(20)_{\text {stat }}\left({ }_{-42}^{+0}\right)_{\text {model }}(7)_{\mathrm{FSE}}(17)_{\text {cutoff }} .
$$

The estimated error due to finite size effects (FSE) is based on [24, 25]. Since the SU(3)-limit $f_{+}^{K \pi}(0)=1$ is exactly obeyed in our simulations cutoff effects can appear only in the difference of the result from one. Indeed, we do not see any indications of cutoff effects in our data (note for example that the pairs of data points corresponding to about 0.1 and 0.15 on the $x$-axis in the plots in figure 1 , respectively, fully agree despite being from lattices with distinct lattice cutoffs). We attach a conservative error of $\left(a \Lambda_{\mathrm{QCD}}\right)^{2} \approx 5 \%$ which is motivated by the automatic $O(a)$-improvement of chiral fermions. This error is then applied to the difference of the result from unity to obtain the uncertainty induced in $f_{+}^{K \pi}(0)$. In figure 3 we compare our result to other lattice computations and results based on chiral perturbation theory and models. Together with $\left|V_{u s} f_{+}^{K \pi}(0)\right|=0.2163(5)$ from experiment [7] we make the prediction $\left|V_{u s}\right|=0.2237\left({ }_{-8}^{+13}\right)$. Supplementing $\left|V_{u d}\right|=0.97425(22)$ [26] and $\left|V_{u b}\right|=4.15(49) \cdot 10^{-3}$ [27], this yields the successful test of CKM first row unitarity, $\left|V_{u d}\right|^{2}+\left|V_{u s}\right|^{2}+\left|V_{u b}\right|^{2}-1=-0.0008\left({ }_{-6}^{+7}\right)$

\section{Analysis with physical light quarks}

In the meantime the RBC/UKQCD collaboration has generated two physical point data sets, $C_{\text {phys }}$ and $A_{\text {phys }}$ and the preliminary results on these ensembles can be seen in the r.h.s. plot in figure 1. While all prior results were obtained from standard correlator computations (i.e. a small number of exact solves on each configurations) these new results were obtained using all-modeaveraging [28] and solves for a large number of source sink separations. This in turn allows us to study in detail the residual contamination of the ground state contributions to correlators by excited states. The r.h.s. plot in figure 3 shows the result for the form factor as a function of the source sink separation (top panel) and the associated statistical uncertainty (bottom panel). We also show a preliminary fit to the dependence of the final result on the source-sink separation which will allow for reducing this source of systematic uncertainty. The r.h.s. plot in figure 1 also shows our preliminary attempts at fitting the data in order to correct for slight mistunings in the light- and strange quark mass. The result (4.2) is compatible with our preliminary new fits. We are currently increasing statistics for the physical point data and will then finalise this analysis. 

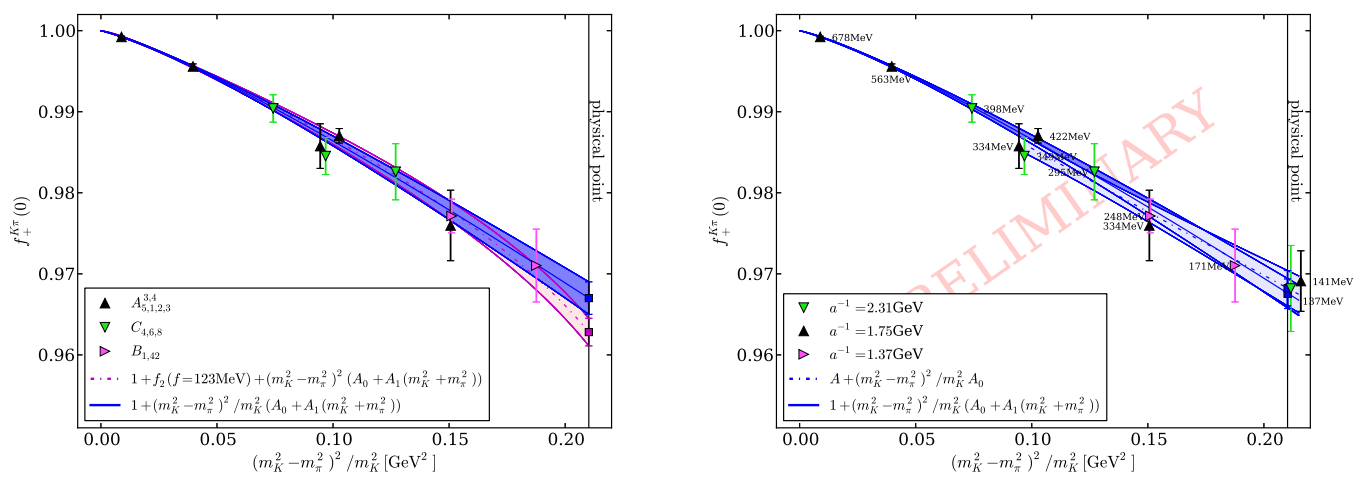

Figure 1: Summary of all simulation data. Left: two ansätze for the mass extrapolation and result at the physical point [5]; Right: two ansätze for the mass extrapolation including the results at the physical point.
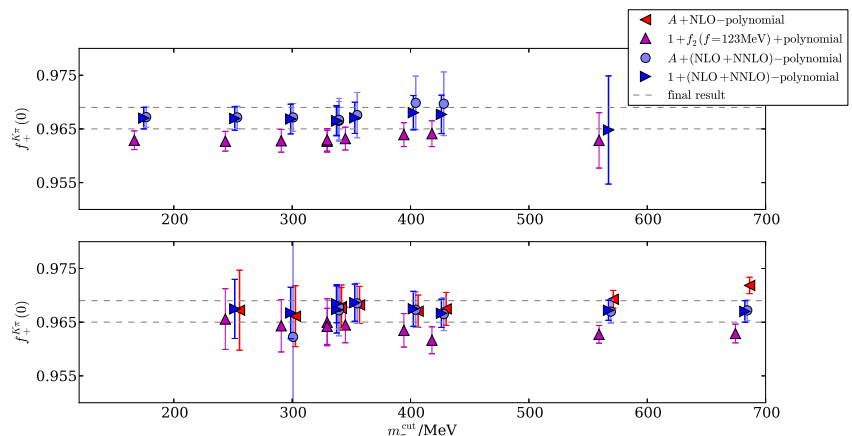

Figure 2: Illustration of the dependence of the results for the form factor on different ansätze under the variation of the lightest (top panel) and heaviest (bottom plot) included pion mass, respectively.
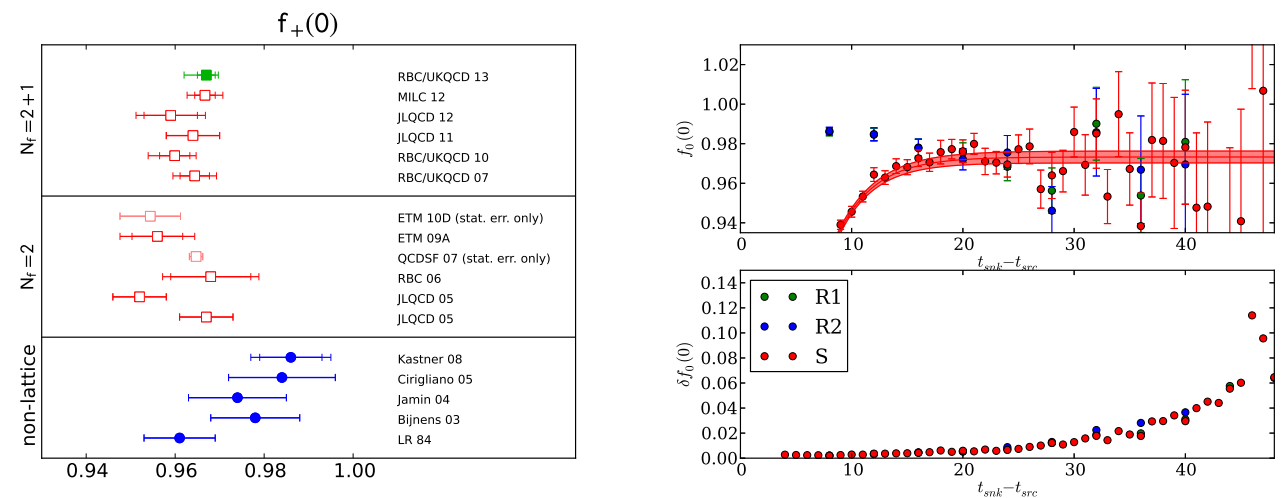

Figure 3: Left: Summary of lattice results for $f_{+}^{K \pi}(0)$. Right: Exponential fit to the dependence of $f_{+}^{K \pi}(0)$ on the source-sink separation. 


\section{Conclusions}

In this talk we have presented the status of the RBC/UKQCD collaborations efforts to compute the $K \rightarrow \pi$ semileptonic form factor at high precision. The objective is a precise first principles determination of the form factor at zero momentum transfer. Together with experimental results this allows for determining the CKM-matrix element $\left|V_{u s}\right|$. We have discussed our fit-strategy and the extension of the published analysis by two new points with physical quark masses. With the help of these two new data points the dominant uncertainty in previous results stemming from the chiral extrapolation will be reduced to a minimum.

Acknowledgements: The research leading to these results has received funding from the European Research Council under the European Union's Seventh Framework Programme (FP7/2007-2013) / ERC Grant agreement 279757. The authors gratefully acknowledge computing time granted through the STFC funded DiRAC facility (grants ST/K005790/1, ST/K005804/1, ST/K000411/1, ST/H008845/1). PAB acknowledges support from STFC Grant ST/J000329/1 and KS is supported by the European Union under the Grant Agreement number 238353 (ITN STRONGnet). Critical to this calculation were the Blue Gene/Q computers at the Argonne Leadership Computing Facility (DOE contract DE-AC02-06CH11357) as well as the RIKEN BNL Research Center and BNL Blue Gene/Q computers at the Brookhaven National Laboratory.

\section{References}

[1] N. Cabibbo, Phys.Rev.Lett. 10 (1963) 531.

[2] M. Kobayashi and T. Maskawa, Prog.Theor.Phys. 49 (1973) 652.

[3] P. Boyle et al., Phys.Rev.Lett. 100 (2008) 141601, 0710.5136.

[4] RBC-UKQCD Collaboration Collaboration, P. Boyle et al., Eur.Phys.J. C69 (2010) 159, 1004.0886.

[5] P.A. Boyle et al., JHEP 1308 (2013) 132, 1305.7217.

[6] G. Colangelo et al., Eur.Phys.J. C71 (2011) 1695, 1011.4408.

[7] M. Antonelli et al., Eur.Phys.J. C69 (2010) 399, 1005.2323.

[8] P.A. Boyle et al., JHEP 05 (2007) 016, hep-lat/0703005.

[9] H. Na et al., Phys.Rev. D82 (2010) 114506, 1008.4562.

[10] P.F. Bedaque and J.W. Chen, Phys.Lett. B616 (2005) 208, hep-lat/0412023.

[11] G. de Divitiis, R. Petronzio and N. Tantalo, Phys.Lett. B595 (2004) 408, hep-lat/0405002.

[12] C. Sachrajda and G. Villadoro, Phys.Lett. B609 (2005) 73, hep-lat/0411033.

[13] RBC-UKQCD Collaboration, B. Mawhinney et al., PoS LATTICE2013 (2013) 404.

[14] Y. Iwasaki and T. Yoshie, Phys. Lett. B143 (1984) 449.

[15] Y. Iwasaki, Nucl. Phys. B258 (1985) 141.

[16] D.B. Kaplan, Phys. Lett. B288 (1992) 342, hep-lat/9206013.

[17] Y. Shamir, Nucl. Phys. B406 (1993) 90, hep-lat/9303005.

[18] R.C. Brower, H. Neff and K. Orginos, Nucl.Phys.Proc.Suppl. 140 (2005) 686, hep-lat/0409118.

[19] R.C. Brower, H. Neff and K. Orginos (2012), 1206.5214. 
[20] RBC Collaboration, UKQCD Collaboration Collaboration, Y. Aoki et al., Phys.Rev. D83 (2011) 074508, 1011.0892 .

[21] RBC-UKQCD Collaboration, C. Allton et al., Phys.Rev. D78 (2008) 114509, arXiv:0804.0473.

[22] J. Gasser and H. Leutwyler, Nucl.Phys. B250 (1985) 517.

[23] J. Bijnens and P. Talavera, Nucl. Phys. B669 (2003) 341, hep-ph/0303103.

[24] K. Ghorbani (2011), 1112.0729.

[25] K. Ghorbani (2013), 1301.0919.

[26] J. Hardy and I. Towner, Phys.Rev. C79 (2009) 055502, 0812.1202.

[27] Particle Data Group Collaboration, J. Beringer et al., Phys.Rev. D86 (2012) 010001.

[28] T. Blum, T. Izubuchi and E. Shintani (2012), 1208.4349.

[29] H. Leutwyler and M. Roos, Z. Phys. C25 (1984) 91.

[30] E. Gamiz et al., JHEP 01 (2003) 060, hep-ph/0212230.

[31] A. Kastner and H. Neufeld, Eur. Phys. J. C57 (2008) 541, 0805.2222.

[32] A. Bazavov et al. (2012), 1212.4993.

[33] JLQCD Collaboration Collaboration, T. Kaneko et al., PoS LATTICE2011 (2011) 284, 1112.5259.

[34] JLQCD Collaboration Collaboration, [JLQCD 12], T. Kaneko et al., PoS LATTICE2012 (2012) 111, 1211.6180.

[35] ETM Collaboration Collaboration, V. Lubicz et al., PoS LATTICE2010 (2010) 316, 1012.3573.

[36] [ETM 09A], B. Blossier et al., JHEP 1004 (2010) 049, 0909.3187.

[37] The QCDSF Collaboration, D. Brommel et al., PoS LAT2007 (2007) 364, arXiv:0710.2100.

[38] C. Dawson et al., Phys. Rev. D74 (2006) 114502, hep-ph/0607162.

[39] JLQCD Collaboration, N. Tsutsui et al., PoS LAT2005 (2006) 357, hep-lat/0510068. 